\title{
Anemia and Associated Factors Among Under Five Children at AsellaTeachingand Referral Hospital, Ethiopia
}

\author{
Bekele Gutema Waye ${ }^{1, *}$, Getahun Megersa ${ }^{2}$ \\ ${ }^{1}$ Department of Medical Laboratory Science, Collage of Health Sciences, Arsi University, Asella, Ethiopia \\ ${ }^{2}$ Department of Medical Laboratory Science, AsellaTeachingand Referral Hospital, Arsi University, Asella, Ethiopia
}

Email address:

Bekishgutema@yahoo.com (B. G. Waye)

${ }^{*}$ Corresponding author

\section{To cite this article:}

Bekele Gutema Waye, Getahun Megersa. Anemia and Associated Factors Among Under Five Children at AsellaTeachingand Referral Hospital, Ethiopia. International Journal of Dental Medicine. Vol. 6, No. 2, 2020, pp. 13-18. doi: 10.11648/j.ijdm.20200602.11

Received: October 5, 2020; Accepted: October 20, 2020; Published: November 23, 2020

\begin{abstract}
Anemia is a condition which results from a reduction in hemoglobin concentration or reduction in red blood cell number or both which resulting in lower ability of oxygen delivery to support the body's activities. It is a wide spread public health problem and has a significant cause of childhood mortality and (WHO) considers anemia prevalence over $40 \%$ as a major public health problem, between 20 and $40 \%$ as a moderate public health problem, and between 5 and $20 \%$ as a mild public health problem. The high prevalence of anemia has its negative consequences for children's health, especially for their growth and development of their mental, physical and social development. It also causes negative behavioral and cognitive effects which resulting in poor school performance and work capacity in later years as different studies indicated. The Objectives of the study is to assess the prevalence of anemia and associated factor among under five children attending pediatrics outpatient department in Asella teaching and referral Hospital June 2018 to September 30 2018. Institutional based cross sectional study was conducted by non- probability convenience sampling technique, 338 children was selected. The results of the study showed that, the overall prevalence of anemia among under five children were $36.3 \%$, around $21(6.2 \%)$ of them were found to be severely anemic, whereas $37(10.9 \%)$ were moderately anemic. Factors like child's age [AOR=2.00, $95 \% \mathrm{CI}(1.15,2.61)]$, birth intervals $[\mathrm{AOR}=2.25,95 \% \mathrm{CI}(1.91,6.94)]$, sex of the children, being stunting $[\mathrm{AOR}=2.50,95 \% \mathrm{CI}$ $(2.00,3.10)]$ and wasting $[\mathrm{AOR}=1.52,95 \% \mathrm{CI}(1.40,3.66)]$, infected with malaria parasites [AOR $=1.12,95 \% \mathrm{CI}(1.00,1.40)]$, were associated with anemia. Anemia remains a common health problem in the study area among under five children and further studies are needed to focus on etiologies and interventions.
\end{abstract}

Keywords: Anemia, Under Five Children, AsellaTeaching, Referral Hospital

\section{Introduction}

Anemia is a decrease in the total amount of red blood cells (RBCs) or hemoglobin in the blood $[1,2]$ or both which result a lowered ability of the blood to carry oxygen [3]. Anemia is major public health problem in developing country among preschool children.

Blood is a body fluid in humans that delivers necessary substances such as nutrients and oxygen to the cells and transports metabolic waste products away from those same cells [4]. It is composed of blood cells suspended in blood plasma. Plasma, which constitutes $55 \%$ of blood fluid, is mostly water ( $92 \%$ by volume). The blood cells are mainly red blood cells (also called RBCs or erythrocytes), white blood cells and platelets [5].

When concentration hemoglobin or red blood cells in the body reduced to below normal anemia developed the most common symptom of all type anemia are include feeling tired, weakness, shortness of breath or a poor ability to exercise. Anemia that comes on quickly often has greater symptoms, which may include confusion, feeling like one is going to pass out, loss of consciousness, or increased thirst. Anemia must be significant before a person becomes noticeably pale. Additional symptoms may occur depending on the underlying cause. [6]

The three main types of anemia are: blood loss, decreased red blood cell production, and increased red blood cell 
breakdown. Causes of blood loss include trauma and gastrointestinal bleeding, among others. Causes of decreased production include iron deficiency, a lack of vitamin B12, thalassemia, and a number of neoplasms of the bone marrow. Causes of increased breakdown include a number of genetic conditions such as sickle cell anemia, infections like malaria, and certain autoimmune diseases [6].

A complete blood count is one of the most frequently requested blood tests to investigation of anemia. There are many potential causes of anemia; the most common in developing country is iron deficiency due to blood loss and anemia of chronic disease. It can be classified into three based on the size of red blood cells and amount of hemoglobin in each cell. If the cells are small, it is microcytic anemia. If they are large, it is macrocytic anemia while if they are normal sized, it is normocytic anemia. Iron deficiency anemia in young children is recognized as a major public health issue and the most prevalent form of micronutrient deficiency worldwide [7-10].

Anemia can be prevented by treating the underlying cause for example; Iron deficiency anemia is treated with oral iron supplements. Patients with uncomplicated iron deficiency can be given a trial treatment with oral iron supplementation to correct anemia and replenish physiological stores [4].

The other prevention mechanism of anemia in child can be restoration of the hemoglobin concentration to normal level by blood transfusion which for patients with iron deficiency anemia are generally only required where there is a risk of cardiovascular instability due to severe anemia, or if patients have symptomatic anemia despite iron treatment. The goal of a transfusion is to restore hemoglobin to a safe, but not necessarily normal, level. [11]

Anemia is a major public health problem in several countries, particularly common among preschool-aged children and women [15]. According to the 2011 World Health Organization (WHO) report, anemia resulting from iron deficiency was one of the most important factors contributing to the global burden of diseases, and it increases morbidity and mortality in preschool-aged children and pregnant women [16].

Anemia is a wide spread public health problem, and it has a significant cause of childhood mortality and (WHO) considers anemia prevalence over $40 \%$ as a major public health problem, between 20 and $40 \%$ as a moderate public health problem, and between 5 and $20 \%$ as a mild public health problem [7].

In sub-Saharan Africa, it is a sever public health problem among preschool-age children. In this region, much of the national prevalence is estimated to be above $40 \%$ among this group [10]. The national level prevalence of anemia in Ethiopia also considered that it is moderate with the prevalence of $(5-44 \%)$; but in some regions of the country high prevalence of anemia was reported. According to the 2016 EDHS report of national level, even if there is intervention the prevalence increased significantly to $72.3 \%$. It means that prevalence of anemia is still at the highest public health problem in Ethiopia. [12]
The high prevalence of anemia has its negative consequences for children's health, especially for their growth and development of their mental, physical and social development [13]. It also causes negative behavioral and cognitive effects which resulting in poor school performance and work capacity in later years as different studies indicated [14].

Hence this study was conducted to assess anemia and associated factor among pre-school age children and the information obtained from the study might be contribute to design effective intervention strategies on study area by concerned body.

\section{Objective}

\subsection{General Objective}

To assess the prevalence of anemia and associated factor among under five children attended pediatrics outpatient department in Asella teaching and referral Hospital from June 2018 to September 2018.

\subsection{Specific Objective}

To assess the prevalence of anemia among under five children in Asella teaching and referral Hospital.

To assess the associated factor of anemia among under five children in Asella teaching and referral Hospital.

\section{Methods and Materials}

\subsection{Study Area}

The study was conducted at Asella teaching and referral hospital. Asella is Located in the Arsi Zone of the Oromia Region about 175 kilometers from Addis Ababa. The town has a latitude and longitude of $7^{\circ} 57^{\prime} \mathrm{N} 39^{\circ} 7^{\prime} \mathrm{E} / 7.950^{\circ} \mathrm{N}$ $39.117^{\circ} \mathrm{E}$, with an elevation of 2,430 meters from sea level. The 2007 national census reported indicate a total population for Asella of 67,269, of whom 33,826 were men and 33,443 were women [15].

Arsi University teaching and referral Hospital currently serves for around 3.5 million populations in Arsi and the nearby zones.

\subsection{Study Design and Period}

Institutional based cross sectional study design was used to assess the magnitude of anemia and associated factor among under-five children in Asella teaching and referral hospital at Asella town, South East Ethiopia from June 2018 to October 2018.

\subsection{Source and Study Population}

The source population was all under five children attending pediatrics outpatient department in Asella teaching and referral hospital during the data collection period.

\subsection{Study Population}

All under five children attending Asella teaching and 
referral hospital pediatrics outpatient department and sent to laboratory for any investigation.

\subsection{Sample Size and Sampling Procedures}

\subsubsection{Sample Size Determination}

The total sample size was determined by using the formula to estimate a single population proportion with the following assumptions: population proportion $(P)$, that is, $72.3 \%$ taken from Ethiopian Demographic Health Survey 2016 report as prevalence of anemia among children aged 6-59 months, 95\% confidence level, and 5\% margin of error. Then, the final sample size 338 was obtained by adding $10 \%$ contingency.

\subsubsection{Sampling Procedure}

Convenient sampling technique was used to recruit a total of 338 children within study time until sample size fulfilled.

Eligible criteria

Inclusion Criteria: - all under five children attended pediatrics outpatient department clinic during study period.

Exclusion Criteria: - under five children who have severely ill.

Variables of the study

Dependent variables

Anemia.

Independent variables

Socio demographic and socio economic of parents like educational level, occupation, place of residence and also age of child's, height of child's, sex of child, weight of child's and MUAC of child, intestinal parasite and hemo-parasites

Operational Definitions

Anemia- is identified by measuring the hemoglobin level $<11$ (g/dl) in blood.

Under five children: - children aged 6-59 months

Mild anemic-Hemoglobin level 10.0-10.9 g/dL

Moderate anemic - hemoglobin level 7-9.9 g/dL whereas and $<7 \mathrm{~g} / \mathrm{dL}$ as severely anemic

Data collection procedures

A structured and standardized questionnaire was used to collect the data. The questioners were prepared in English language by principal investigator and then translated to local language.

One day training was given for data collectors before the actual data collection, furthermore a pre-test of the instrument and the procedure was conducted and corrective measures were taken.

Hemoglobin (Hgb) concentration was determined by Sysmex fully automated CBC analyzer from blood sample. Venus blood was used, sample was collected from arm after rubbing the site with sterile cotton (immersed in $70 \%$ alcohol), and collected around $3 \mathrm{ml}$ of blood in EDTA tube. Blood films was prepared and stained with Giemsa stain for the assessment of hemo-parasites.

In addition to the laboratory investigation to determine anemia, the height, MUAC and weight of children were measured by Nurses and BMI calculated to determine malnutrition status of child and also stunting and wasting of child was calculated by using WHO anthroplus application

Data quality assurance

The quality of data was controlled starting from the time of questionnaires preparations. The questionnaires were developed by reviewing relevant literatures on the subject to ensure reliability.

Quality of laboratory test result also checked by running high, medium and low quality control material daily and blood film quality also checked starting from smear preparation and reagent quality check up to have quality smear. Standard operating procedure (SOPs) was followed during laboratory analysis

After the completion of the data collection, the questionnaire was checked for its completeness, unrecorded values and unlikely responses were manually clean up on such indication. The test result was written on the laboratory data collection format sheet

Data analysis method

Data entry and analyses was done by SPSS version 21 programs to calculate frequencies and percentile of each variable that describes participant characteristics. Significance level and association of variables was tested by using 95\% confidence interval (C. I) and odd ratio. P-value less than 0.05 were taken as statistically significant.

Ethical considerations

Ethical clearance was obtained from department of medical laboratory Sciences and ethical review and research committee. Official letters were taken from the Arsi University College of health science to Asella teaching and referral Hospital and pediatrics Department.

Oral consents were taken from parents/care giver after creating awareness about objective, benefit of the study, individual's right.

Any participants who are not volunteers were not enforced to include as study subject and they can stop or refuse to study participant any moment they want. At the end they are informed the result of them. Participant who is anemic were communicated with principal investigator and help them on getting necessary treatment.

\section{Result}

\subsection{Socioeconomic and Demographic Characteristics of a Family}

A total of 338 children aged 6-59 months with their mothers/care giver were included in the study with the response rate of $100 \%$. Thirty seven percents $126(37.3 \%)$ of mothers/care giver attended their education only up to elementary school and around $83(26.3 \%)$ mothers of participant attended higher education (diploma and above). Regarding paternal education, $46.7 \%$ were attended their education diploma and above.

Twenty two percents of participants' family had lowest monthly income which was less than 1000 Ethiopian Birr, whereas the majorities were around $56.5 \%$ had more than 3000.00 Birr income per month (table 1). 
Table 1. Sociodemographic of participants' family in Asella teaching and referral Hospital Asella, Oromia, Ethiopia, October 2018 ( $n=338)$.

\begin{tabular}{lll}
\hline Variable & Frequency & Percent (\%) \\
\hline Resident of child family & & \\
Urban & 171 & 50.6 \\
Rural & 167 & 49.4 \\
Education status of Father & & \\
Illiterate & 9 & 2.7 \\
Elementary & 76 & 22.5 \\
Secondary & 96 & 28.4 \\
Diploma and above & 157 & 46.4 \\
Education status of Mother & & \\
Illiterate & 25 & 7.4 \\
Elementary & 126 & 37.3 \\
Secondary & 104 & 30.8 \\
Diploma and above & 83 & 24.6 \\
Occupation of Father & & \\
Merchant & 52 & 15.4 \\
Farmer & 136 & 40.2 \\
Civil servant & 150 & 44.4 \\
Occupation of Mother & & \\
Housewife & 201 & 59.5 \\
Merchant & 71 & 21 \\
Civil servant & 66 & 19.5 \\
Monthly income of family & 76 & 22.5 \\
$<1000$ & 11 & 3.3 \\
1000-2000 & 60 & 17.8 \\
2000-3000 & 71 & 21 \\
3000-4000 & 61 & 18 \\
$4000-5000$ & 59 & 17.5 \\
$>5000$ & & \\
Birth interval of mother & 73 & 78.4 \\
$<2$ year & 265 & \\
$>$ 2 year & & \\
\hline & & \\
& &
\end{tabular}

\subsection{Demographic Characteristics and Nutritional Status of Children}

Out of 338 study participants, 155 (45.9\%) were males and $183(36.4 \%)$ were females. Majority of the children were 611 months old. Regarding durations of breast feeding, only $80(23 \%)$ were found with history of breast feed until 2 year (table 2).

Table 2. Demographic Characteristics of under five children in Asella teaching and referral Hospital Asella, Oromia, Ethiopia, October 2018 $(n=338)$.

\begin{tabular}{lll}
\hline Variable & Frequency & Percent (\%) \\
\hline Age & & \\
6 Month & 91 & 26.9 \\
6-11 Month & 83 & 24.6 \\
12-23 month & 63 & 18.6 \\
24-59 month & 101 & 29.9 \\
Sex of child & & \\
Male & 155 & 45.9 \\
Female & 183 & 54.1 \\
Malnutrition status of child & & \\
Normal & 141 & 41.7 \\
Mild malnutrition & 160 & 47.3 \\
Severe malnutrition & 37 & 10.9 \\
Body mass index of child & & \\
Normal & 119 & 35.2 \\
Under weight & 211 & 62.4 \\
Obese & 8 & 2.4 \\
Total time of Brest feed of child & & \\
6 only month & 64 & 18.9 \\
\hline
\end{tabular}

\begin{tabular}{lll}
\hline Variable & Frequency & Percent (\%) \\
\hline up to 1 year & 194 & 57.4 \\
up to 2 year & 80 & 23.7 \\
\hline
\end{tabular}

\subsection{Magnitude of Anemia among Children}

Anemia was measured using the hemoglobin level of the child. The over all of anemia among the children were 124 (36.7\%). The mean hemoglobin level was about $11.96(+/-$ $2.96) \mathrm{g} / \mathrm{dl}$ which ranged from $2 \mathrm{~g} / \mathrm{dl}$ to $18.0 \mathrm{~g} / \mathrm{dl}$. Accordingly, more than two-third of the children, $214(63.3 \%)$, were non anemic and around $21(6.2 \%)$ of them were found to be severely anemic, whereas $37(10.9 \%)$ were moderately anemic (table 3).

Furthermore, it also differs by child age category. A children aged 6-11 month were more anemic than the other which was $45.8 \%$ and prevalence relatively decreased in children the aged 24-59 months.

Table 3. The degree of anemia among children aged 6-59 months based on Hgb level in Asella referral and teaching Hospital Asella, Oromia, Ethiopia, $2018(n=338)$.

\begin{tabular}{lll}
\hline Variable & Frequency & Percent (\%) \\
\hline Anemia status based on Hgb level & & \\
Not anemic & 214 & 63.3 \\
Mild anemic & 66 & 19.5 \\
Moderate anemic & 37 & 10.9 \\
Sever anemic & 21 & 6.2 \\
\hline
\end{tabular}

\section{Discussion}

This study was assessed the prevalence of anemia and factors associated with it among children aged 6-59 months. The overall prevalence of anemia was found to be $36.7 \%$ which was relatively lower than the national prevalence of 44\% Ethiopian 2011 DHS report and EDHS 2016 report (72.3\%). Our study result slightly less than prevalence of anemia in sub-Saharan Africa $42 \%$ and more less than Swaziland (91\%) [16]. and it was consistence to the prevalence of Tigray region (37.5\%) [9]. whereas lower than Amhara Region (42\%) [16]. the variation might be due to the difference in geographical locations and socio economic variations of the study area.

According to cross tabulation of age category of children with anemia status majorities were in6-11month age group which was $45.8 \%$ which followed with $24-59$ month age group (27\%). Children with previous medical illness before this study were much more anemic than those had no medical illness $72.9 \%$ from total of 48 children.

Prevalence of anemia was higher among malnourished children than normal which $82.3 \%$. Child with underweight (low BMI) had also anemic than the normal. Regarding anemia status among sex of the children females are higher anemic than male which was $45.9 \%$ from a total of 183 females and $25 \%$ from 155 total children respectively (table 4).

Regarding factors Associated with Anemia: Different parental, childhood, environmental and household variables were tested for their association with the presence of anemia 
among under-five children by binary logistic regression analysis. First the association of each variable with the occurrence of anemia among under-five children was assessed by binary logistic regression, and then variables which $\mathrm{P}<0.25$ were entered into the final model to control confounding. Multivariate logistic regression analysis was done to assess independent association of the factors with anemia. Accordingly, children of age 6-11month were two times more likely hood of developing anemia than those of children 24-59 months. Sex of the children also show statistically significant association with anemia which female children were more protective $0.39(0.24,0.63)$ than male children's (table 4).

Being stunting and wasting were more likely hood of developing anemia than normal children where 2.5 (2.00, $3.10)$ and $2.00(1.90,3.66)$ respectively.

Generally, children's age, birth intervals, sex of the children, being stunting and wasting, infected with malaria parasites were associated with anemia (Table 4).

Table 4. Associated factors anemia among children aged 6-59 months in Asella referral and teaching Hospital Asella, Oromia, Ethiopia, 2018 (n=338).

\begin{tabular}{|c|c|c|c|}
\hline \multirow{2}{*}{ Variables } & \multicolumn{3}{|l|}{ Anemic status } \\
\hline & Number (\%) & Crude OR (95\% CI) & Adjusted OR (95\% CI) \\
\hline \multicolumn{4}{|c|}{ Age of child in month } \\
\hline 6 & $91(26.9)$ & $1.73(0.93,3.15)$ & $1.50(1.17,1.68)$ \\
\hline $6-11$ & $83(24.5)$ & $2.31(1.24,4.58)$ & $2.0(1.15,2.61)$ \\
\hline $12-23$ & $63(18.5)$ & $1.576(1.20,3.0)$ & $1.0(1.25,2.35)$ \\
\hline $24-59$ & $101(29.9)$ & 1 & 1 \\
\hline \multicolumn{4}{|c|}{ Sex of child } \\
\hline Female & $155(45.9)$ & $0.39(0.24,0.63)$ & $0.42(0.25,0.72)$ \\
\hline Male & $183(54.1)$ & 1 & 1 \\
\hline \multicolumn{4}{|c|}{ MUAC of the child } \\
\hline$>11 \mathrm{~cm}$ & $141(41.7)$ & $0.21(0.13,0.36)$ & $0.24(0.14,0.43)$ \\
\hline$<12 \mathrm{~cm}$ & $197(58.3)$ & 1 & 1 \\
\hline \multicolumn{4}{|l|}{ Stunting } \\
\hline Yes & $132(39.1)$ & $2.9(2.20,3.50)$ & $2.5(2.00,3.10)$ \\
\hline No & $206(60.9)$ & 1 & 1 \\
\hline \multicolumn{4}{|l|}{ Wasting } \\
\hline Yes & $184(54.4)$ & $2.05(1.67,2.64)$ & $2.00(1.90,3.66)$ \\
\hline No & $154(45.6)$ & 1 & 1 \\
\hline \multicolumn{4}{|c|}{ Birth interval of family } \\
\hline$<2$ year & $73(21.6)$ & $3.65(1.56,4.51)$ & $2.25(1.91,6.94)$ \\
\hline$>2$ year & $265(78.4)$ & 1 & 1 \\
\hline \multicolumn{4}{|c|}{ Malaria positive } \\
\hline No & $330(41.7)$ & 1 & 1 \\
\hline Yes & $8(47.3)$ & $1.15(1.08,1.31)$ & $1.12(1.00,1.40)$ \\
\hline
\end{tabular}

\section{Conclusion and Recommendation}

Anemia was found to be a moderate public health problem (36.7\%) among children aged 6-59 months attended in Asella referral and teaching hospital. It was established that occurrence of anemia is directly correlated with parents' income and maternal literacy status. Childs' age, birth intervals, sex of the children, being stunting and wasting, infected with malaria parasites were associated with anemia.

Therefore, appropriate and tailored interventional strategies are required to reduce the prevalence of childhood anemia. These include improving providing health education on child Brest feeding practices and complementary food and also birth interval of child must be a jested until child start other complementary food other than Brest milk only.

\section{Conflict of Interests}

The authors declare that they have no competing interests.

\section{Acknowledgements}

The authors would like to express their sincere gratitude to those children and their mother/caretaker for their willingness and positive cooperation for being part of the study.

\section{References}

[1] World Health Organization. Worldwide prevalence of anemia 1993-2005: WHO global database on anemia. Edited by Bruno de Benoist, Erin McLean, Ines Egli and Mary Cogswell, Geneva, 2008.

[2] United Nations Administrative Committee on Coordination Nutrition (ACC/ SCN) 4TH Report on the world nutrition situation: Nutrition throughout the Life Cycle. Sub-Committee on Geneva: ACC/SCN; 2000.

[3] Schellenberg D, Schellenberg JRMA, Muchi A, et al. The silent burden of anemia in Tanzanian children: a communitybased study. Bulletin of the World Health Organization 2003; 81: 581-590. 
[4] Cornet M, Le Hesran JY, Fievet N, et al. Prevalence of and risk factors for anemia in young children in southern Cameroon. Am J Trop Med Hyg 1998; 58: 606-611

[5] Micronutrient Initiative/UNICEF. Vitamin and mineral deficiency: A global progress report. Ottawa, 2004. Available at: $\quad$ http://micronutrient.org/CMFiles/PubLib/VMd-GPREnglish1KWW-3242008-4681.pdf.

[6] Adish A, Esrey S, Gyorkos T, Johns T. Risk factors for iron deficiency anemia in preschool children in Northern Ethiopia. Public Health Nutr 1999; 2: 243-252.

[7] WHO. Iron Deficiency Anaemia: Assessment, Prevention, and Control. A guide for programmer managers. Geneva: World Heal Organ; 2001. 114

[8] Benoist B de, McLean E, Egll I, Cogs well M. Worldwide prevalence of anemia 1993-2005. Geneva: WHO Global Database on Anemia; 2008. VI+41pp.

[9] Mclean E, Cogswell M, Egli I, Wojdyla D, De Benoist B. Worldwide prevalence of anemia, WHO vitamin and mineral nutrition information system, 1993-2005. Public Health Nutr. 2009; 12 (4): 444-54.

[10] R. J. S. Magalhaes, "Archie CA Clements: spatial variation in childhood anemia in Africa," Bulletin of the World Health Organization, vol. 89, pp. 459-468, 2011.

[11] Cape Verde. Ministerio da Saude. Inquerito Demografico e de Saude Reprodutiva (IDSR) 2005. Praia: Instituto Nacional de
Estatistica, Ministerio da Saude, Republica De Cabo Verde, 2008. 312 p. (http://www.ine.cv/actualise/publicacao March 2008).

[12] C. S. A. Ethiopia Mini Demographic and Health Survey 2016 Key Indicators Report. Addis Ababa, Ethiopia 2016 [Accessed: 01 Feb 2017].

[13] Grantham-McGregor S, Ani C: A review of studies on the effect of iron deficiency on cognitive development in children. J Nutr 2001, 131 (2): 649-666.

[14] Nokes C, van den Bosch C, Bundy DAP: The Effects of Iron Deficiency and Anemia on Mental and Motor Performance, Educational Achievement, and Behaviors in Children. An Annotated Bibliography. Washington, DC: INACG and ILSI Press; 1998

[15] Central Statistical Agency [Ethiopia] and ORC Macro, Ethiopia Demographic and Health Survey 2011, Central Statistical Agency, Addis Ababa, Ethiopia; ORC Macro, Calverton, Md, USA, 2011.

[16] A Steel N. Global, regional, and national age-sex specific mortality for 264 causes of death, 1980-2016: a systematic analysis for the global burden of disease study 2016. Lancet. 2017; 390 (10100): 1151-210.

[17] Central Statistical Agency. Ethiopia Demographic and Health Survey 2011. Addis Ababa, Ethiopia; 2012. Available at: https://dhsprogram.com/pubs/pdf/FR255/FR255.pdf. Accessed date: $18 / 10 / 2017$. 